\title{
Disturbance Observer-Based Complementary Fractional-Order Sliding Mode Control for PMSM Drive System
}

\author{
Yong-Hong Lan $(\mathbb{D}$, Li-Tao Zheng, and Zhao-Hong Wang \\ School of Automation and Electronic Information, Xiangtan University, Xiangtan, Hunan 411105, China \\ Correspondence should be addressed to Yong-Hong Lan; 961501563@qq.com
}

Received 19 August 2020; Revised 21 October 2020; Accepted 23 November 2020; Published 3 December 2020

Academic Editor: Chih-Chiang Chen

Copyright (c) 2020 Yong-Hong Lan et al. This is an open access article distributed under the Creative Commons Attribution License, which permits unrestricted use, distribution, and reproduction in any medium, provided the original work is properly cited.

\begin{abstract}
In this paper, a disturbance observer-based complementary fractional-order sliding mode control (CFOSMC) scheme is proposed for the permanent magnet synchronous motor (PMSM) drive system. First, to reconstruct the load disturbance and parameter variations, a nonlinear disturbance observer is designed. Next, a disturbance observer-based fractional-order sliding mode with a saturation function control law is designed to reduce the chattering problem in the existing fractional-order sliding mode control (FOSMC) method. Furthermore, to reduce the thickness of the boundary layer, a CFOSMC scheme is designed. By using the fractional-order Lyapunov stability theorem, the existence condition and the chattering problem are analyzed. Compared with the existing FOSMC, the obtained CFOSMC law does not contain any high-order derivatives of tracking error, which is easier to implement. Finally, the numerical simulations and experimental results are provided to show the superiority of the proposed method. To improve the performance of the permanent magnet synchronous motor (PMSM) drive system in terms of tracking rapidity, accuracy, and robustness, a complementary fractional-order sliding mode control (CFOSMC) scheme with disturbance observer is proposed in this paper.
\end{abstract}

\section{Introduction}

Permanent magnet synchronous motor (PMSM) has many applications in industries due to its excellent features such as superpower density, high torque to current ratio, fast response, and low noise $[1,2]$. However, the PMSM is a typical multivariable coupled high nonlinear system, and its performance is sensitive to external load disturbances and parameter uncertainties. Over the last decades, various design methods have been developed, such as robust control [3], predictive control [4], adaptive control [5], and neural network control [6].

Recently, to improve the control performances in PMSM diver systems, much attention has been given to the disturbance observer-based (DOB) control method. In [7], a DOB state feedback controller was designed for the PMSM system. On the basis of this paper, a sensorless control method for PMSM drive was developed in [8]. In [9], a generalized predictive current control method combined with sliding mode disturbance compensation was proposed to satisfy the requirement of fast response and strong robustness. By combining the adaptive sliding mode control with sliding mode disturbance observer, a hybrid control strategy was proposed in [10]. In [11], a new estimation method of sensor faults and unknown disturbance in current measurement circuits for the PMSM drive system was presented. However, the above-proposed DOB control schemes only focus on the load torque disturbance but lack the estimation of parameter uncertainties.

To counteract the disturbances and uncertainties, sliding mode control (SMC) is a powerful nonlinear control technique, which has been widely used for speed and position control of the PMSM system [12]. To estimate the immeasurable mechanical parameters of PMSM, an intelligent second-order SMC using a wavelet fuzzy neural network estimator was proposed in [13]. Considering the time-varying characteristic and the high-bandwidth property of the uncertainties and disturbances in a PMSM drive 
system, a disturbance observer-based SMC scheme was introduced in [14]. To estimate the online stator resistance, a new sliding mode observer for sensorless SMC was presented in [15]. To further improve the performance of the SMC, a robust SMC scheme based on a rapid nonlinear tracking differentiator was proposed in [16]. The secondorder SMC design problem was discussed in [17], in which a novel saturation function was used. Based on the conventional SMC, adding a generalized error sliding surface, the complementary sliding mode control (CSMC) was proposed for the fault-tolerant control of a six-phase PMSM drive system in [18]. The complementary sliding mode control method based on Elman neural network was proposed in [19], which can not only reduce the system state to the sliding surface time but also guarantee the system tracking accuracy. Considering that the control system in a PMSM has uncertainties and disturbances, in [20], an adaptive switching gain was proposed. Comparisons of experimental results show that the proposed method has a faster adjustment process than SMC. In [21], a disturbance observerbased CSMC design method was proposed for the PMSM control system of the mine traction electric locomotive.

As we all know, one obvious disadvantage of the SMC method is the chattering phenomenon caused by discontinuous control law and frequent switching action near the sliding surface. Besides, the upper bound of lumped disturbances is not easy to be determined, which could worsen the chattering phenomenon of SMC strategy. Recently, to overcome these drawbacks, some fractional-order sliding mode control (FOSMC) schemes have been widely adopted to weaken the chattering phenomenon and deal with external disturbances. An FOSMC scheme based on parameters auto-tuning for the velocity control of PMSM was proposed in [22]. A robust FOSMC was proposed for the position control of PMSM in [23]. By selecting a proper fractional-order and designing a fractional-order sliding surface, the proposed FOSMC is distinctly more excellent than that of the conventional SMC. To investigate the position regulation problem of PMSM subject to parameter uncertainties and external disturbances, an FOSMC was proposed and the finite-time stability of the closed-loop system was obtained in [24]. Based on the fractional stability theory, a sliding mode control scheme for synchronization of fractional PMSM was developed in [25]. A novel FOSMC for a class of integer-order systems with mismatched disturbances was proposed in [26]. In [27], a disturbance observer-based composite supertwisting sliding mode control was designed for the PMSM speed regulation problem. To improve the convergence rate over the existing sliding mode control method for the trajectory tracking control, an adaptive fast nonsingular integral terminal SMC method was proposed in $[28,29]$.

Note that the above SMC-based control methods for the PMSM driver system can deal with mismatched disturbance and have better control performance with faster response speed, lower overshoot, and less chattering effect than the traditional control strategy. However, the high-order derivative of the tracking error (or the reference signal) was usually used in the sliding model controller [19, 20, 22]. From a practical point of view, it is difficult to implement. On the other hand, there is still room for improvement when it comes to the chattering phenomenon in classic FOSMC. This observation motivates our current study. In this paper, we mainly investigate a modified FOSMC scheme with a load disturbance observer for the speed control of PMSM. The main contributions are as follows: (1) to estimate the load disturbance and parameter uncertainties, a nonlinear disturbance observer is constructed and the asymptotic stability condition for observation error is also obtained; (2) to reduce the chattering phenomenon in the existing FOSMC method, a disturbance observer-based fractional-order sliding mode with saturation function control law is designed; and (3) to reduce the thickness of boundary layer, a CFOSMC law with disturbance observer is proposed. The tracking performance and robustness of the proposed method are also analyzed and compared with the conventional FOSMC scheme.

The rest of this paper is organized as follows: in Section 2, the mathematic model of PMSM and problem formulation are presented. The nonlinear disturbance observer design and stability analysis are derived in Section 3. In Section 4, the conventional FOSMC for the PMSM drive system is improved and the disturbance observer-based CFOSMC method is derived. The effectiveness of the proposed algorithm is illustrated in Section 5 through numerical simulations and experiment examples. Finally, some conclusions are drawn in Section 6.

\section{Mathematical Model of PMSM and Problem Formulation}

The mathematics model of a PMSM can be described in the rotor rotating reference frame as follows $[10,22,23]$ :

$$
\left\{\begin{array}{l}
u_{q}^{*}=R_{s} i_{q}^{*}+\dot{\lambda}_{q}+\omega_{f} \lambda_{d}, \\
u_{d}^{*}=R_{s} i_{d}^{*}+\dot{\lambda}_{d} q+\omega_{f} \lambda_{q}, \\
\lambda_{q}=L_{q} i_{q}^{*}, \\
\lambda_{d}=L_{d} i_{d}^{*}+L_{m} I_{\mathrm{df}}, \\
\omega_{f}=n_{p} \omega_{r}^{*},
\end{array}\right.
$$

where $u_{d}^{*}, u_{q}^{*}$ are the $d, q$-axis stator voltages; $i_{d}^{*}, i_{q}^{*}$ are the $d, q$-axis stator currents; $\lambda_{d}, \lambda_{q}$ are the $d, q$-axis stator flux linkages; and $L_{d}, L_{q}$ are the $d, q$-axis stator inductances. While $\omega_{f}$ and $\omega_{r}^{*}$ are the inverter frequency and rotor speed, respectively, $L_{m}$ is the $d$-axis mutual inductance; $I_{\mathrm{df}}$ is the equivalent $d$-axis magnetizing current; $n_{p}$ is the number of pole pairs; and $R_{s}$ is the stator resistance.

The electric torque is stated as

$$
T_{e}=3 n_{p} \frac{\left[L_{m} I_{\mathrm{df}} i_{q}^{*}+\left(L_{d}-L_{q}\right) i_{q}^{*} i_{d}^{*}\right]}{2} .
$$

Motor dynamics is presented as

$$
T_{e}=J \dot{\omega}_{r}+B_{m} \omega_{r}+T_{l},
$$

where $T_{l}$ is the load torque, $B_{m}$ is the viscous friction coefficient, and $J$ is the moment of inertia. 
By using the field-oriented mechanism with $i_{d}=0$ $[10,22]$, we can simplify the electric torque as

$$
T_{e}=k_{p}^{*} i_{q}^{*}=\frac{3 n_{p} L_{m} I_{\mathrm{df}}}{2} i_{q}^{*} .
$$

Substituting (3) into (4), one can obtain the state equation of servo drive:

$$
\left\{\begin{array}{l}
\dot{\omega}_{r}=-a \omega_{r}+b i_{q}-c, \\
a=\frac{B_{m}}{J}, b=\frac{k_{p}^{*}}{J}, c=\frac{T_{l}}{J} .
\end{array}\right.
$$

Considering the uncertainties and time-invariant parameters, we can rewrite (5) as

$$
\dot{\omega}_{r}=-(a+\Delta a) \omega_{r}+(b+\Delta b) i_{q}-(c+\Delta c),
$$

where $\Delta a, \Delta b, \Delta c$ are the time-invariant parameters.

The tracking error $e(t)$, in terms of the desired reference speed $\omega_{r}^{*}(t)$ and the measured actual output speed $\omega_{r}(t)$, is defined as

$$
e(t)=\omega_{r}^{*}(t)-\omega_{r}(t)
$$

The time derivative of $e(t)$ is

$$
\left\{\begin{array}{l}
\dot{e}(t)=-a e(t)-b i_{q}(t)+\phi(t)+d, \\
\phi(t)=a \omega_{r}^{*}(t)+c+\dot{\omega}_{r}^{*}(t), \\
d=\Delta a \omega_{r}(t)-\Delta b i_{q}(t)+\Delta c
\end{array}\right.
$$

where $d$ is time-variant uncertainties such as mechanical parameter variations, friction force, load disturbances, speed distortion, and current harmonics. It is assumed that

$$
|d| \leq \Lambda(>0), \lim _{t \longrightarrow \infty} \dot{d}=d^{*},
$$

where $d^{*}$ is a constant.

In this paper, the control objective is to find a suitable FOSMC input $i_{q}^{*}(t)$ such that the output speed $\omega_{r}(t)$ can track the desired reference speed $\omega_{r}^{*}(t)$ asymptotically in the presence of any arbitrary initial conditions and uncertainties.

\section{Disturbance Observer Design}

To estimate time-variant lumped uncertainties and load torque disturbance, we use the following nonlinear disturbance observer [30]:

$$
\left\{\begin{array}{l}
\dot{p}(t)=-l \widehat{d}+l\left[a e(t)+b i_{q}(t)-\phi(t)\right], \\
\widehat{d}=p(t)+l e(t)
\end{array}\right.
$$

where $\widehat{d}$ is the estimation of $d$ and $l>0$ is the observer gain.

Estimation error of the disturbance observer is defined as

$$
\widetilde{d}=\widehat{d}-d,
$$

and error dynamics of disturbance observer is governed by

$$
\dot{\vec{d}}=\dot{\hat{d}}-\dot{d} \text {. }
$$

It can be derived from (10)-(12) that

$$
\dot{\tilde{d}}=-\tilde{l d}-\dot{d}
$$

Since $\quad l>0$ and $\lim _{t \rightarrow \infty} \dot{d}=d^{*}$, we have $\lim _{t \rightarrow \infty} \tilde{d}=-\left(d^{*} / l\right)$.

Now, we give the following result.

Lemma 1. For nonlinear system (8), suppose that the disturbance observer is formulated as (10); then, the disturbance estimation error $\tilde{d}$ is bounded.

Remark 1. It follows from Lemma 1 that the observer gain $l$ can change the bound of the disturbance estimation error. Clearly, it should be selected as large enough.

\section{Disturbance Observer-Based CFOSMC Scheme Design}

Considering that the disturbance observer (10) can estimate the load torque disturbance accurately, we will replace $d$ with $\widehat{d}$ in the following synthesis.

4.1. Traditional FOSMC. Fractional calculus is a generalization of integer-order integration and differentiation to noninteger-order ones. Let $\mathscr{D}^{\alpha}$ denote the fractional-order derivative, which is defined as in Definition 1.

Definition 1 (see [31]). The Caputo derivative is defined by

$$
\mathscr{D}^{\alpha} f(t)=\frac{1}{\Gamma(n-\alpha)} \int_{t_{0}}^{t}(t-\tau)^{-\alpha+n-1} f^{(n)}(\tau) \mathrm{d} \tau,
$$

where $n$ is the first integer which is not less than $\alpha$, that is, $\alpha \in[n-1, n) . \Gamma(\cdot)$ is the well-known Gamma function, which is defined by $\Gamma(z)=\int_{0}^{\infty} e^{-z} t^{z-1} \mathrm{~d} t$.

Definition 2 (see [31]). The definition of the fractional integral is described by

$$
\mathscr{D}^{-\alpha} f(t)=\frac{1}{\Gamma(\alpha)} \int_{t_{0}}^{t}(t-\tau)^{\alpha-1} f(\tau) \mathrm{d} \tau, \quad \alpha>0 .
$$

To proceed with the discussion, the following lemmas will be used.

Lemma 2 (see [32]). Autonomous system is as follows:

$$
\mathscr{D}^{\alpha} x(t)=A x(t),
$$

with $x\left(t_{0}\right)=x_{0}$ and $0<\alpha<1$, is asymptotically stable if and only if $|\arg (\operatorname{spec}(A))|>(\alpha \pi / 2)$, where $\operatorname{spec}(A)$ is the spectrum (set of all eigenvalues) of $A$. Also, the state vector $x(t)$ decays towards 0 and meets the condition $\|x(t)\|<M t^{-\alpha}, t>0, M>0$.

Lemma 3 (see [33]). Let $x(t) \in \mathbb{R}^{n}$ be a vector of a differentiable function. Then, for any time instant $t \geq t_{0}$, the following relationship holds: 


$$
\frac{1}{2} \mathscr{D}^{\alpha}\left(x^{T}(t) P x(t)\right) \leq x^{T}(t) P \mathscr{D}^{\alpha} x(t), \quad \forall \alpha \in(0,1],
$$

where $P \in \mathbb{R}^{n \times n}$ is a positive definite matrix.

Lemma 4 (see [34]). Let $x(t)=0$ be an equilibrium point for the nonautonomous fractional-order system $\mathscr{D}^{\alpha} x(t)=f(x(t), t)$, where $f(x(t), t)$ is locally Lipschitz in $x(t)$. Assume that there exists a Lyapunov candidate $V(x(t), t)$ satisfying

$$
\begin{aligned}
\alpha_{1}\|x(t)\|_{2}^{a} & \leq V(x(t), t) \leq \alpha_{2}\|x(t)\|_{2}^{a b}, \\
\mathscr{D}^{\beta} V(x(t), t) & \leq-\alpha_{3}\|x(t)\|_{2}^{a b},
\end{aligned}
$$

where $\alpha_{1}, \alpha_{2}, \alpha_{3}, a$, and $b$ are positive constants and $\beta \in(0,1)$. Then, the equilibrium point is Mittag-Leffler stable.

Remark 2 Mittag-Leffler stability implies asymptotic stability [34].

Clearly, the fractional-order operator has more degrees of freedom than that with integer order. It is likely that a better performance can be obtained with the proper choice of order [22, 23].

As presented in $[22,23]$, a traditional fractional-order sliding surface $S$ can be chosen as

$$
S=\lambda e(t)+\mathscr{D}^{\alpha} e(t)
$$

where $\lambda>0$ and $0<\alpha<1$.

Taking the time derivative on both sides of (19) and using $\widehat{d}$ to estimate $d$, we have

$$
\begin{aligned}
\dot{S} & =\lambda \dot{e}(t)+\mathscr{D}^{\alpha+1} e(t) \\
& =\lambda\left(-a e(t)-b i_{q}(t)+\phi(t)+\widehat{d}\right)+\mathscr{D}^{\alpha+1} e(t) .
\end{aligned}
$$

The equivalent control law can be selected as

$$
i_{q}=u_{\mathrm{eq}}=\frac{1}{\lambda b}\left(-a \lambda e(t)+\lambda \phi(t)+\lambda \widehat{d}+\mathscr{D}^{\alpha+1} e(t)\right) .
$$

As usual, we adopt the following approach law [22]:

$$
\dot{S}=\varepsilon S+\rho \operatorname{sgn}(S),
$$

where $\varepsilon>0, \rho>0$, and $\operatorname{sgn}(\cdot)$ denotes the sign function, which is defined as

$$
\operatorname{sgn}(S)= \begin{cases}1, & S \geq 0, \\ 0, & S=0, \\ -1, & S<0 .\end{cases}
$$

From (21) and (22), the final controller can be designed as

$$
\begin{aligned}
i_{q}= & u_{\mathrm{eq}}+u_{v} \\
= & u_{\mathrm{eq}}+\varepsilon S+\rho \operatorname{sgn}(S) \\
= & \frac{1}{\lambda b}\left(-a e(t)+\phi(t)+\widehat{d}+\mathscr{D}^{\alpha+1} e\right) \\
& +\varepsilon S+\rho \operatorname{sgn}(S) .
\end{aligned}
$$

When the sliding mode occurs, system (19) can be represented as

$$
\mathscr{D}^{\alpha} e(t)=-\lambda e(t) \text {. }
$$

Thus, by Lemma 2, system (25) is asymptotic stable.

Remark 3. As pointed out in Lemma 2, the state $e(t)$ of the fractional-order system (25) decays towards 0 like $t^{-\alpha}$. But, in the case of the integer-order system, it decays towards 0 like $e^{-t}$. It means that the energy transfer is slower with fractional-order sliding surface than that with integer-order one $[22,23]$. Therefore, the fractional-order sliding surface is smoother compared with the integer-order one. As a result, the chattering can be better attenuated with a fractionalorder controller.

Remark 4. It is worth pointing out that if $d=0$, then the fractional-order sliding mode controller (24) is reduced to

$$
\begin{aligned}
i_{q}= & u_{\mathrm{eq}}+u_{v} \\
= & u_{\mathrm{eq}}+\varepsilon S+\rho \operatorname{sgn}(S) \\
= & \frac{1}{\lambda b}\left(-a e(t)+\phi(t)+\mathscr{D}^{\alpha+1} e\right) \\
& +\varepsilon S+\rho \operatorname{sgn}(S),
\end{aligned}
$$

which is the same as the one proposed in [22].

It can be seen that the $\operatorname{sgn}(\cdot)$ function is involved in (26). As pointed out in [23], the chattering phenomenon will be caused. In the following, a saturation function sat $(\cdot)$ is adopted to further reduce the chattering problem, which is described as follows:

$$
\operatorname{sat}(S)= \begin{cases}1, & S \geq \Phi, \\ \frac{S}{\Phi}, & -\Phi<S<\Phi, \\ -1, & S \leq-\Phi,\end{cases}
$$

where $\Phi>0$ denotes the thickness of the boundary layer. Clearly, when the saturation function is used, the final controller (24) can be modified as

$$
\begin{aligned}
i_{q}= & \frac{1}{\lambda b}\left(-a e(t)+\phi(t)+\widehat{d}+\mathscr{D}^{\alpha+1} e(t)\right) \\
& +\varepsilon S+\rho \operatorname{sat}(S) .
\end{aligned}
$$

The Lyapunov function is defined as

$$
V(t)=\frac{1}{2} S^{2}
$$

Calculating the derivation of (29) and invoking (20) and (28), it yields 


$$
\begin{aligned}
\dot{V}(t) & =S \dot{S} \\
& =S\left(\lambda \dot{e}(t)+\mathscr{D}^{\alpha+1} e(t)\right) \\
& =S \lambda\left(-a e(t)-b i_{q}(t)+\phi(t)+\widehat{d}\right)+\mathscr{D}^{\alpha+1} e \\
& =-\varepsilon S^{2}-S \operatorname{sat}\left(\frac{S}{\Phi}\right) \leq 0 .
\end{aligned}
$$

According to the Lyapunov stability theorem, the reaching condition of the sliding mode controller is satisfied, which indicates that the system will converge to the switching manifold asymptotically.

Remark 5. Note that the high-order derivative $\mathscr{D}^{\alpha+1} e$ is used in fractional-order sliding model controller (24) and (28). From a practical point of view, it is difficult to implement.

Remark 6. From (19) and (27), it can be seen that the sliding mode surface $S$ will finally maintain on the steady state of $|S| \leq \Phi$. That is, the closed-loop system is in a steady state with $|e(t)| \leq(\Phi / \lambda)$. Though the large $\Phi$ can reduce the chattering mostly, it also can increase the ultimate bound of the tracking error $e(t)$. To trade off the chattering and control performance, conservative $\lambda$ and $\Phi$ are always selected by trial and error.

4.2. Disturbance Observer-Based CFOSMC. As stated in Remark 6, there exists a boundary layer $\Phi$ of $S$ in a steady state. In this state, the boundary layer is also the tracking error boundary layer for a given $\lambda$. In this section, to further reduce the thickness of the boundary layer, a complementary fractional-order sliding mode controller is designed.

Borrowed from [18-20], the complementary fractionalorder sliding mode surface is defined as

$$
\bar{S}=S_{g}+S_{c},
$$

where

$$
\begin{aligned}
& S_{g}=\mathscr{D}^{\alpha} e(t)+2 \lambda e(t)+\lambda^{2} \mathscr{D}^{-\alpha} e(t), \quad \lambda>0, \\
& S_{c}=\mathscr{D}^{\alpha} e(t)-\lambda^{2} \mathscr{D}^{-\alpha} e(t), \quad \lambda>0 .
\end{aligned}
$$

Taking the $\alpha$ order time derivation on both sides of (32) and (33), respectively, we have

$$
\begin{aligned}
& \mathscr{D}^{\alpha} S_{g}=\mathscr{D}^{2 \alpha} e(t)+2 \lambda \mathscr{D}^{\alpha} e(t)+\lambda^{2} e(t), \\
& \mathscr{D}^{\alpha} S_{c}=\mathscr{D}^{2 \alpha} e(t)-\lambda^{2} e(t) .
\end{aligned}
$$

From (31) to (35), we can obtain

$$
\begin{gathered}
\bar{S}=S_{g}+S_{c}=2\left(\mathscr{D}^{\alpha} e(t)+\lambda e(t)\right), \\
\mathscr{D}^{\alpha} S_{g}-\mathscr{D}^{\alpha} S_{c}=2 \lambda\left(\mathscr{D}^{\alpha} e(t)+\lambda e(t)\right)=\lambda \bar{S} .
\end{gathered}
$$

The Lyapunov function candidate is chosen as

$$
V_{c}(t)=\frac{1}{2}\left(S_{g}^{2}+S_{c}^{2}\right) .
$$

Taking the $\alpha$ order derivative of (38) and using Lemma 3 as well as (32) to (37), we have

$$
\begin{aligned}
\mathscr{D}^{\alpha} V_{c}(t) \leq & S_{g} \mathscr{D}^{\alpha} S_{g}+S_{c} \mathscr{D}^{\alpha} S_{c} \\
\leq & S_{g} \mathscr{D}^{\alpha} S_{g}+S_{c}\left(\mathscr{D}^{\alpha} S_{g}-\lambda S_{c}\right) \\
= & \left(S_{g}+S_{c}\right)\left(\mathscr{D}^{\alpha} S_{g}-\lambda S_{c}\right) \\
= & \bar{S}\left(\mathscr{D}^{2 \alpha} e(t)+2 \lambda \mathscr{D}^{\alpha} e(t)+\lambda^{2} e(t)-\lambda S_{c}\right) \\
= & \bar{S}\left(\mathscr{D}^{2 \alpha-1} \dot{e}(t)+2 \lambda \mathscr{D}^{\alpha} e(t)+\lambda^{2} e(t)-\lambda S_{c}\right) \\
= & \bar{S}\left[\mathscr{D}^{2 \alpha-1}\left(-a e(t)-b i_{q}(t)+\phi(t)+\widehat{d}(t)\right)\right. \\
& \left.+2 \lambda \mathscr{D}^{\alpha} e(t)+\lambda^{2} e(t)-\lambda S_{c}\right] .
\end{aligned}
$$

Therefore, if the complementary fractional-order sliding mode controller is designed as

$$
i_{q}=i_{\mathrm{eq}}+i_{v}
$$

where

$$
\begin{aligned}
i_{\mathrm{eq}}= & \frac{1}{b}\left[(-a e(t)+\phi(t)+\widehat{d}(t))+2 \lambda \mathscr{D}^{1-\alpha} e(t)\right. \\
& \left.+\lambda^{2} \mathscr{D}^{1-2 \alpha} e(t)-\lambda \mathscr{D}^{1-2 \alpha} S_{c}\right] \\
i_{v}= & \frac{\rho}{b} \mathscr{D}^{1-2 \alpha} \text { sat }(\bar{S}), \quad \rho>0,
\end{aligned}
$$

then

$$
\mathscr{D}^{\alpha} V_{c}(t) \leq-\rho \bar{S} \operatorname{sat}(\bar{S}) \leq 0,
$$

which means that $\bar{S}$ will approach zero in a finite-time duration and the system is globally stable.

Remark 7. Note that the CFOSMC scheme (41) can be rewritten as

$$
\begin{aligned}
i_{\mathrm{eq}}= & \frac{1}{b}\left[(-a e(t)+\phi(t)+\hat{d}(t))+2 \lambda \mathscr{D}^{1-\alpha} e(t)\right. \\
& \left.+\lambda^{2} \mathscr{D}^{1-2 \alpha} e(t)-\lambda\left(\mathscr{D}^{1-\alpha} e(t)-\lambda^{2} \mathscr{D}^{1-3 \alpha} e(t)\right)\right] .
\end{aligned}
$$

Since $0<\alpha<1$, the highest order derivative of $e(t)$ in controller (44) is less than 1 , which is easier to implement in practice than FOSMC (24) and (28).

Remark 8. When $(1 / 3)<\alpha<1$, the fractional-order differentiation of $e(t)$ is contained in controller (44). It can be viewed as a low-pass filter and reduce the amplitude of highfrequency fluctuations of $e(t)$ [23]. When $\alpha=(1 / 2)$, especially, controller (44) is a fractional-order $P I^{(1 / 2)} D^{(1 / 2)}$ controller. As we all know, fractional-order PID is a trade-off between higher precision (provided by a higher order of integrator) and stability (provided by a higher order of 


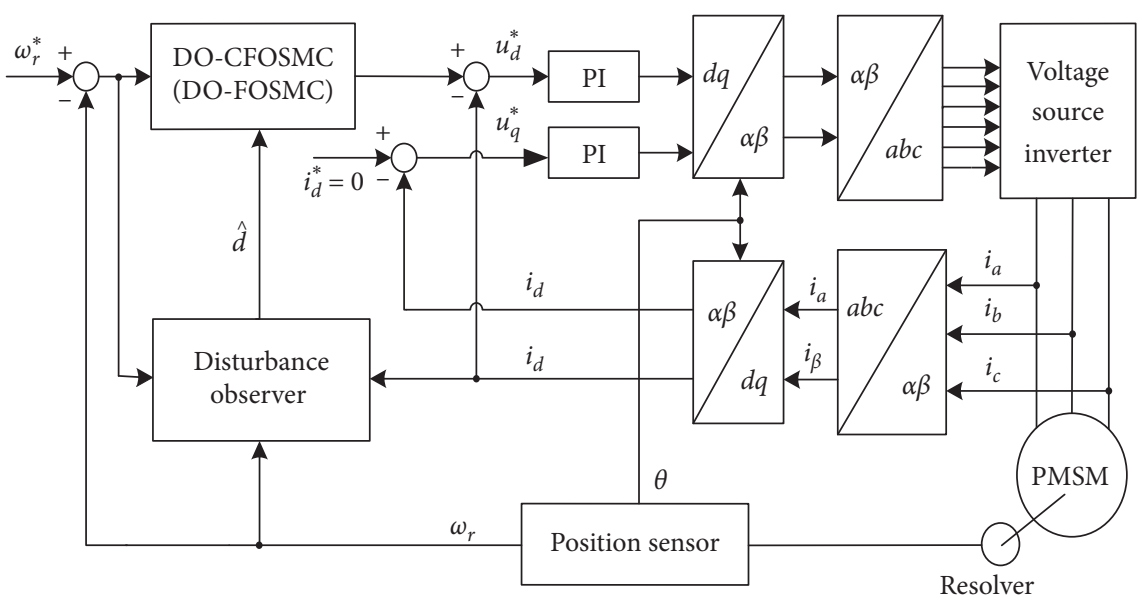

Figure 1: Block diagram of the PMSM position control system.

differentiator). Hence, CFOSMC (41) is more smoother than the conventional FOSMC (24) and (28). On the other hand, there are several parameters in the fractional-order sliding mode controller (42). As we all know, one of the difficulties in SMC-based controller design is the determination of the controller parameters. In this paper, we obtained them by trial and error. In fact, even with the effort and knowledge of the control designer, it can not guarantee that the set of parameters is optimal.

Remark 9. When the system is in a steady state, the sliding surface function $\bar{S}$ will finally maintain on the state of $|\bar{S}| \leq \Phi$. From (27) and (36), we can see that the closed-loop system is in a steady state with $|e(t)| \leq(\Phi / 2 \lambda)$. Compared with disturbance observer-based FOSMC with saturation function, CFOSMC (41) can eliminate the chattering problem further.

\section{Simulations and Experiments}

In this section, the numerical example and experimental results are presented to demonstrate the validity of the proposed method.

5.1. Numerical Simulation Results. The configuration of the overall simulation control system is shown in Figure 1, in which DO-FOSMC means the disturbance based FOSMC with saturation function (controller (28)), and DOCFOSMC is the proposed disturbance observer-based CFOSMC (controller (40)). The performances of the proposed DO-CFOSMC are analyzed and compared with that of DO-FOSMC.

The parameters of the PMSM system are set as follows: rated power $P=10 \mathrm{KW}$, pole pairs is $p=3$, friction factor is $B=0.001(\mathrm{~N} \cdot \mathrm{m} \cdot \mathrm{s} / \mathrm{rad})$, stator inductance is $L=0.0153 \mathrm{H}$, rotor moment of inertia is $J=0.0021 \mathrm{~kg} \cdot \mathrm{m}^{2}$, permanent magnet flux is $\phi_{f}=0.82 \mathrm{wb}$, and stator resistance is $R=0.56 \Omega$. The parameters of DO-CFOSMC and DOFOSMC are selected as $l=5, \Phi=0.01, \lambda=8000, \rho=3000$. From the simulation results, we found that the best selection range for the fractional-order of the proposed FOSMC method is $\alpha \in\left[\begin{array}{ll}0.50 & 0.60\end{array}\right]$. In the following, we set $\alpha=0.50$.

The initial rotation speed of the motor is given as $r_{f 1}=1200 \mathrm{r} / \mathrm{min}$. When $t=0.6 \mathrm{~s}$, the rotation speed is set to $r_{f 2}=1000 \mathrm{r} / \mathrm{min}$. The initial load torque of the motor is $0 \mathrm{~N} \cdot \mathrm{m}$ while $t=0.8 \mathrm{~s}$, and the load torque is $10 \mathrm{~N} \cdot \mathrm{m}$, which can be viewed as an external disturbance.

The numerical simulation results are shown in Figures 2-4. Figure 2(a) indicates the actual speed responses of the proposed DO-CFOSMC and conventional DOFOSMC in the presence of the above load disturbance. Figure 2(b) shows the rotation speed response when the PMSM motor just started. In the time $t=0.6 \mathrm{~s}$, the reference rotation speed has a sudden change. The rotation speed response is depicted in Figure 2(c). Figure 2(d) shows the rotation speed response at $t=0.8 \mathrm{~s}$, in this time, the external disturbance load is added suddenly. From Figure 2, it can be seen that the output can both track precisely the reference input signal. But, under the DO-FOSMC method, the speed response is faster and more stable, whereas the chatter amplitude occurring in conventional DO-FOSMC is bigger than that in DO-CFOSMC.

With respect to the DO-FOSMC method, the threephase current of the stator is shown in Figure 3. The current frequency is inversely proportional to the rotation speed, while the current amplitude is proportional to the rotation torque and changes rapidly as the load torque varies. The variation of electromagnetic torque is shown in Figure 4 as the speed and load torque change. It can be indicated that the motor has a faster and small overshoot torque response with the DO-CFOSMC than that in DO-FOSMC.

In order to evaluate the robustness of the proposed DOCFOSMC scheme under parameter variations, numerical simulations are also carried out. The tested PMSM electric parameters are the mutual inductance $L_{m}=0.0158$, viscous friction coefficient $f_{m}=0.006$ and stator resistance $R_{m}=0.75$. The parameters of the controllers (DO-CFOSMC and DO-FOSMC) are set the same as before.

Figure 5 shows a comparison of the speed responses between the proposed DO-CFOSMC and conventional DOFOSMC methods under parameter variations. It is observed 


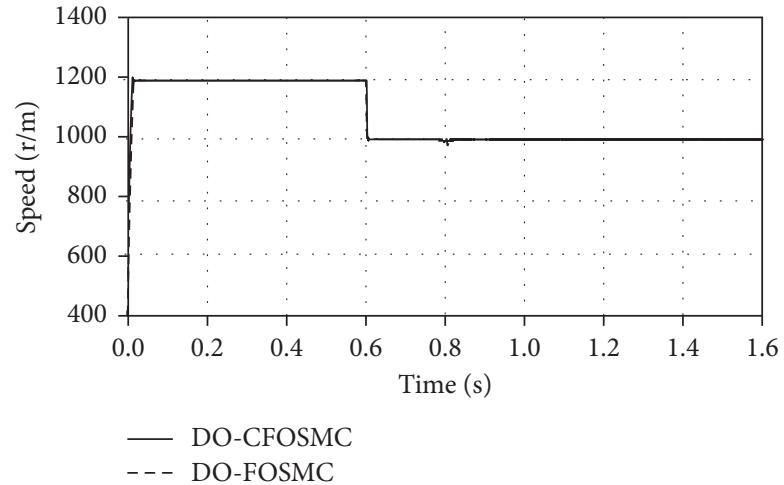

(a)

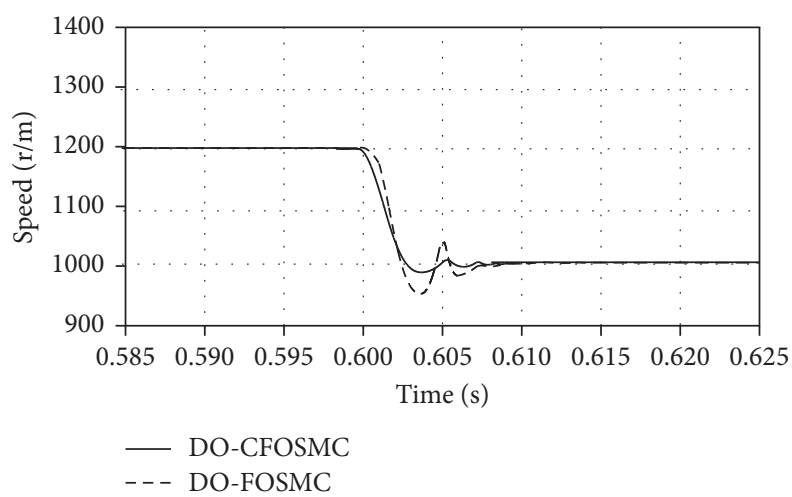

(c)

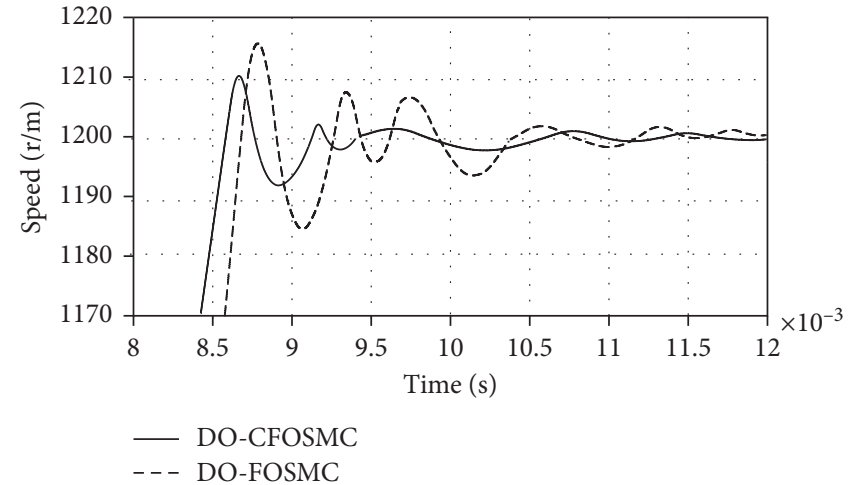

(b)

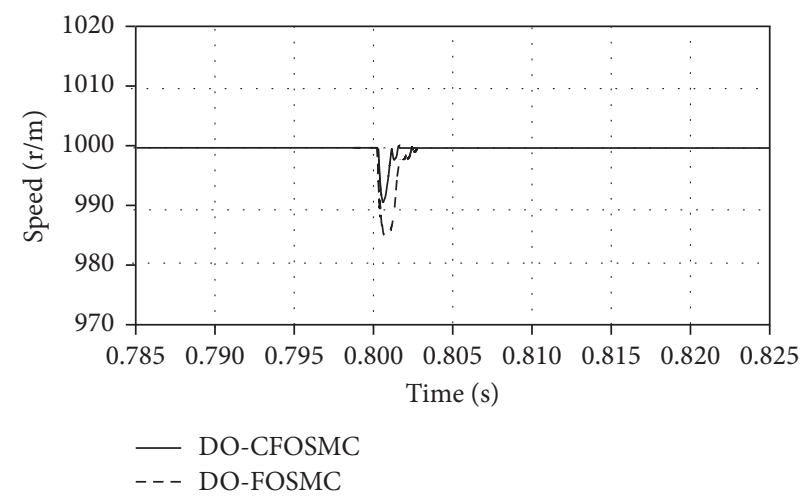

(d)

FIGURE 2: Rotation speed responses.

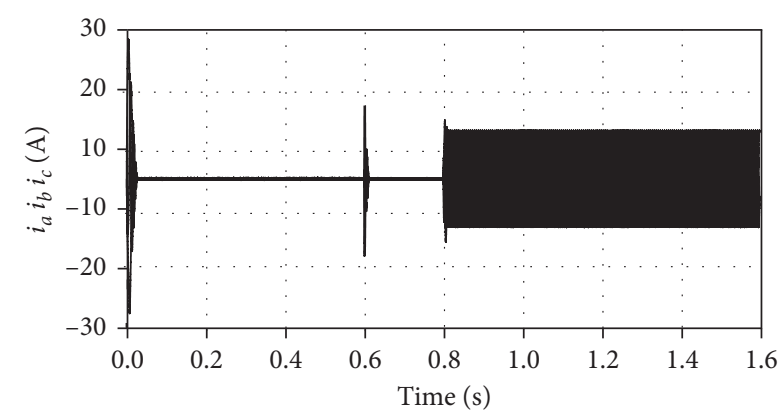

FIGURE 3: Three-phase stator current.

that both controllers can make the output speed track the desired reference speed accurately. But the DO-CFOSMC can realize the closed-loop system more robust to parameter variations and guarantee a better tracking performance.

According to the above numerical simulations, the effectiveness of the proposed DO-CFOSMC scheme with respect to the rejection of external disturbance and parameter variations has been verified. The results show that the proposed DO-CFOSMC scheme gives better tracking performances than that of the conventional DO-FOSMC.

5.2. Experimental Results. To further investigate the effectiveness of the proposed control scheme, some real-time experiments are carried out. The PMSM speed control

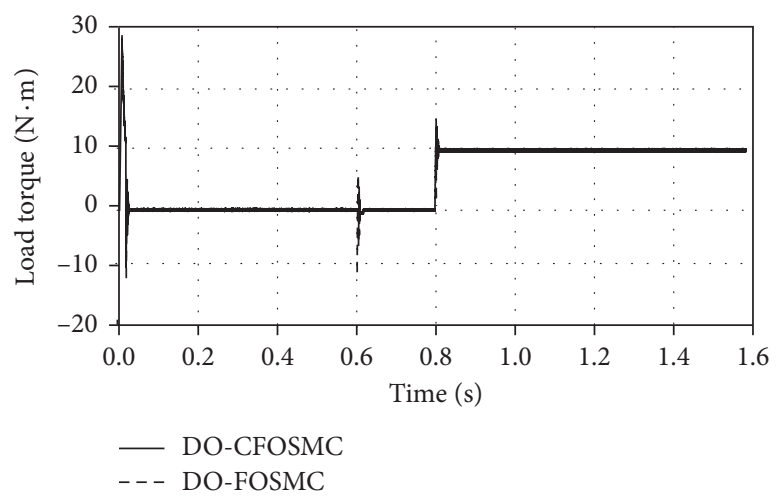

Figure 4: Load torque responses.

platform is illustrated in Figure 6. The main chip of the inverter adopts the TMS320F28335 digital signal processor (DSP).

The initial rotation speed of the motor is $0 \mathrm{r} / \mathrm{min}$, and the rotation speed is $800 \mathrm{r} / \mathrm{min}$ at $4 \mathrm{~s}$. The load torque of the motor is $0 \mathrm{Nm}$. Figures 7(a) and 7(b) show the dynamic responses of the speed and torque, respectively. From Figure 7(a), it can be seen that the DO-CFOSMC method has a smaller overshoot and a shorter settling time than that of the conventional DOFOSMC method. Figure 7(b) shows that when the PMSM motor just started, the maximum load torque under the DO- 


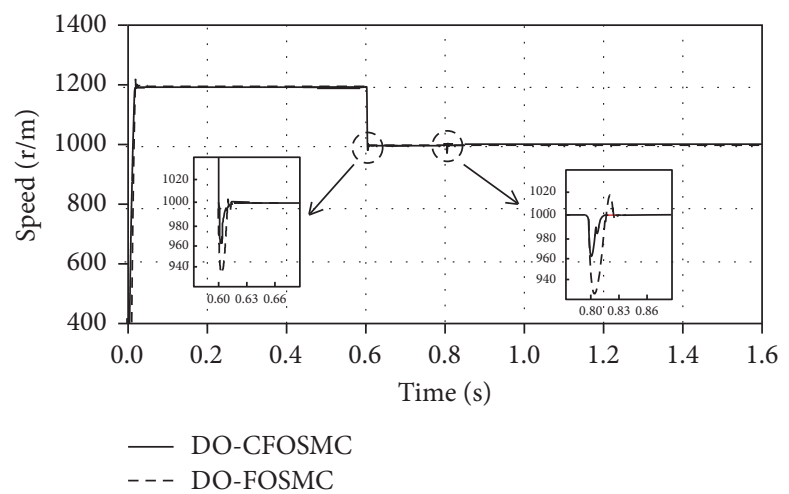

FIGURE 5: Rotation speed responses under parameter variations.

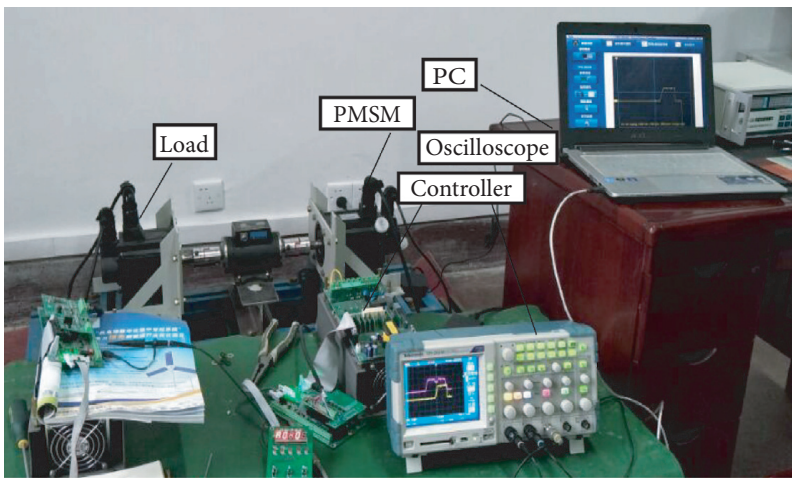

FIGURE 6: PMSM speed control platform.

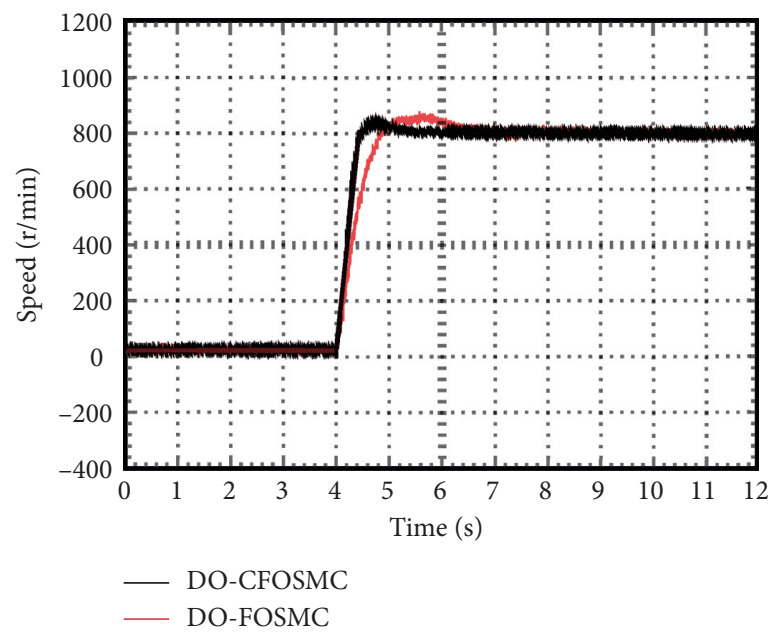

(a)

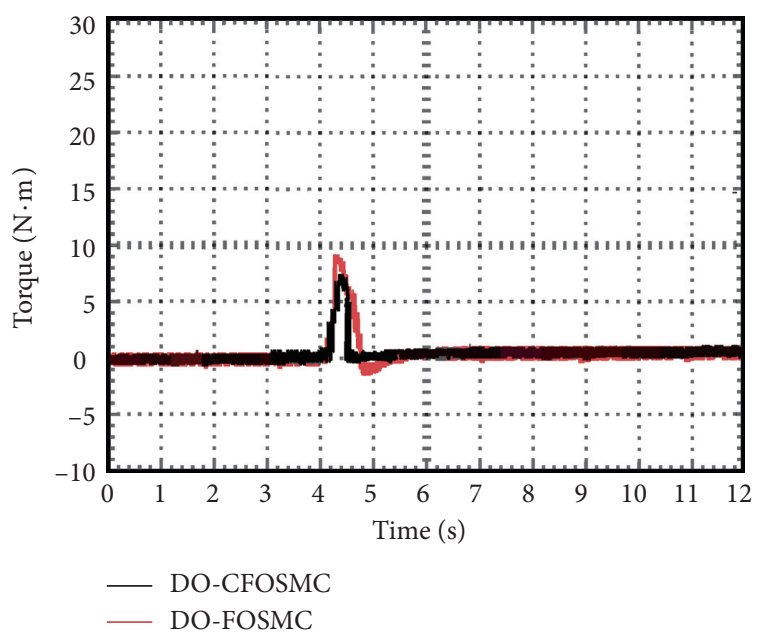

(b)

FIGURE 7: Experimental results of the startup procedure.

FOSMC method is $9 \mathrm{Nm}$, whereas the DO-CFOSMC method reduces it to $7 \mathrm{Nm}$. Moreover, the adjustment time required for the load torque to return to its original value decreases obviously with the DO-CFOSMC method.

When a load disturbance torque $T_{l}=14 \mathrm{Nm}$ is added and removed suddenly at $0.8 \mathrm{~s}$ and $1.2 \mathrm{~s}$, respectively, the dynamic responses of the speed and torque are shown in
Figures 8 and 9, respectively. From these figures, we can see that, under the DO-CFOSMC method, the fluctuation maximum values of the speed and torque are smaller, while the recovering time against load disturbance is shorter than that of the conventional DO-FOSMC method.

The above experimental results show that the DOCFOSMC method achieves a smaller fluctuation 


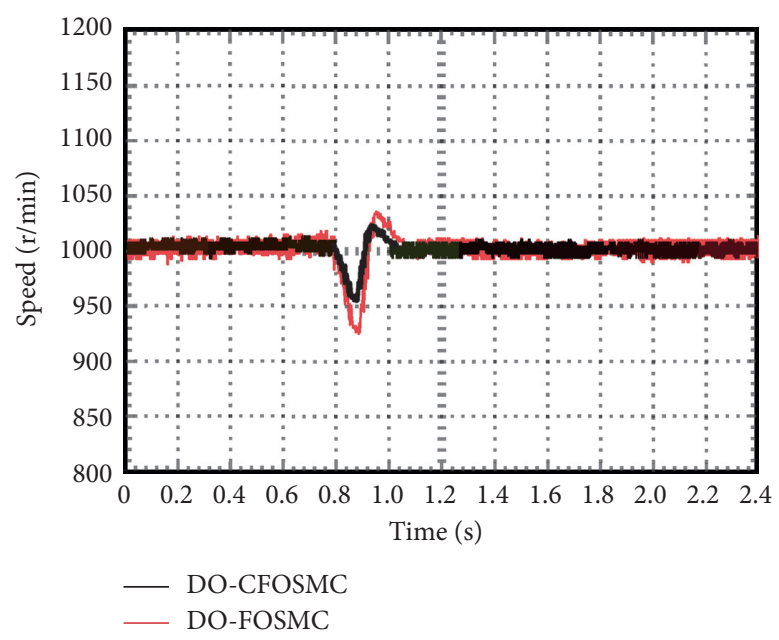

(a)

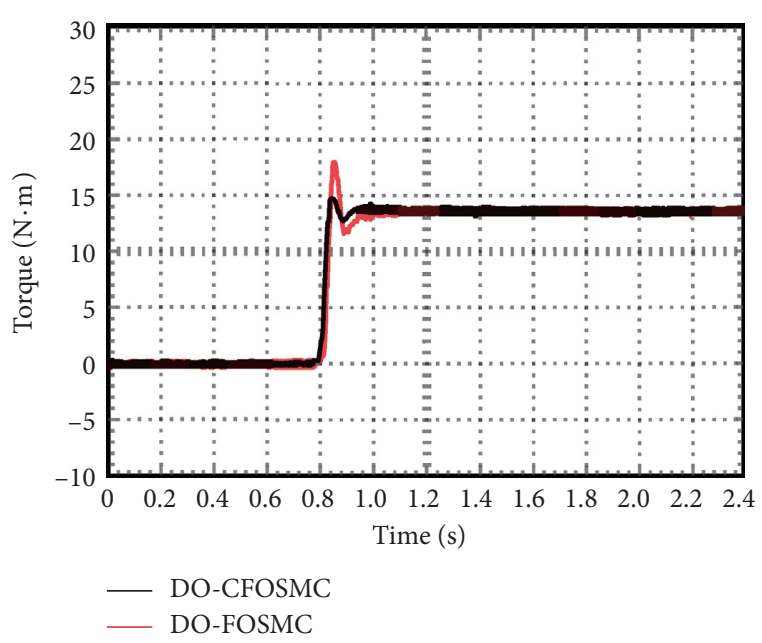

(b)

FIgURE 8: Experimental results of adding sudden load torque.

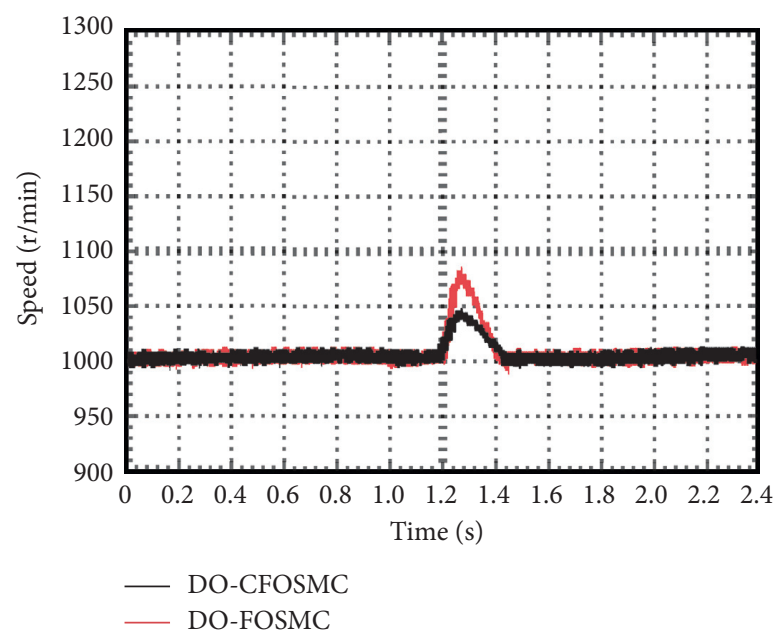

(a)

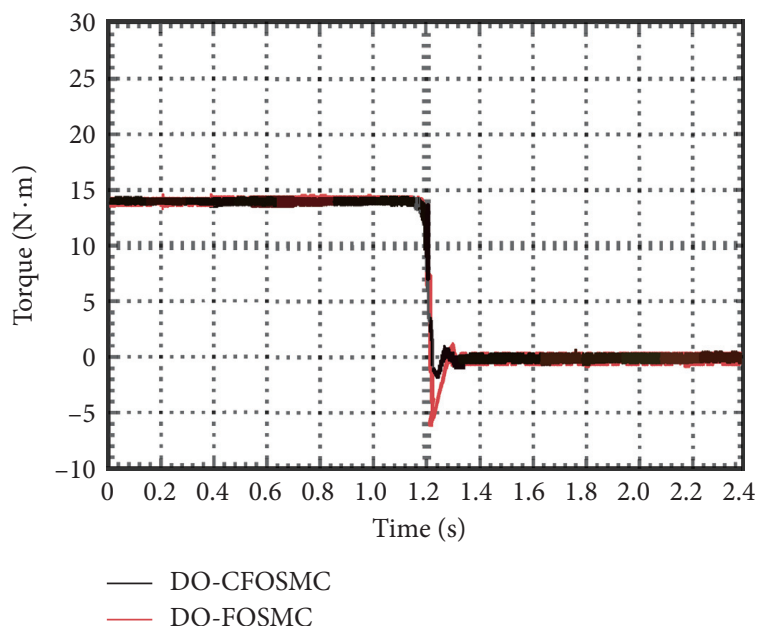

(b)

FiguRE 9: Experimental results of removing sudden load torque.

overshoot with respect to the rotation speed response. In addition, the DO-CFOSMC method guarantees that the adjustment time required for the load torque to return to its original value is smaller than that with the DO-FOSMC method. Therefore, the proposed DO-CFOSMC scheme exhibits a satisfactory tracking rapidity, accuracy, and robustness.

\section{Conclusion}

In this paper, in order to improve the antidisturbance capability of the PMSM drive system, a disturbance observer-based CFOSMC method has been presented, in which a nonlinear observer was employed to estimate the model uncertainties and load disturbance, while the CFOSMC scheme was utilized to improve the performance of the PMSM in terms of the tracking rapidity, accuracy, and robustness. Both numerical simulations and experimental results have shown the effectiveness of the proposed method. Our future work includes adaptive nonsingular integral terminal CFOSMC with disturbance observer for PMSM. Moreover, the fuzzy logic inference scheme to tune the gains of switching control law will also be included.

\section{Data Availability}

No data were used to support this study.

\section{Conflicts of Interest}

The authors declare that they have no conflicts of interest. 


\section{Acknowledgments}

This work was supported in part by the National Natural Science Foundation of PR China (61573298) and Hunan Provincial Natural Science Foundation of China (2020JJ6037).

\section{References}

[1] S. Bolognani, R. Oboe, and M. Zigliotto, "Sensorless fulldigital PMSM drive with EKF estimation of speed and rotor position," IEEE Transactions on Industrial Electronics, vol. 46, no. 1, pp. 184-191, 1999.

[2] A. Djerioui, A. Houari, M. Ait-Ahmed, M.-F. Benkhoris, A. Chouder, and M. Machmoum, "Grey Wolf based control for speed ripple reduction at low speed operation of PMSM drives," ISA Transactions, vol. 74, pp. 111-119, 2018.

[3] S. Mandra, K. Galkowski, and H. Aschemann, "Robust guaranteed cost ILC with dynamic feedforward and disturbance compensation for accurate PMSM position control," Control Engineering Practice, vol. 65, pp. 36-47, 2017.

[4] T. Turker, U. Buyukkeles, and A. F. Bakan, "A robust predictive current controller for PMSM drives," IEEE Transactions on Industrial Electronics, vol. 63, no. 6, pp. 3906-3914, 2016.

[5] J. Chen, W. Yao, Y. Ren, R. Wang, L. Zhang, and L. Jiang, "Nonlinear adaptive speed control of a permanent magnet synchronous motor: a perturbation estimation approach," Control Engineering Practice, vol. 85, pp. 163-175, 2019.

[6] S. Gao, H. Dong, B. Ning, T. Tang, and Y. Li, "Nonlinear mapping-based feedback technique of dynamic surface control for the chaotic PMSM using neural approximation and parameter identification," IET Control Theory \& Applications, vol. 12, no. 6, pp. 819-827, 2018.

[7] A. A. Aishwarya, A. J. Vrunda, A. W. Rahee, and A. A. Godbole, "Speed control of PMSM using disturbance observer," IFAC-papers OnLine, vol. 49, no. 1, pp. 308-314, 2016.

[8] X. Lu, H. Lin, and J. Han, "Load disturbance observer-based control method for sensorless PMSM drive," IET Electric Power Applications, vol. 10, no. 8, pp. 735-743, 2016.

[9] X. Liu, C. Zhang, K. Li, and Q. Zhang, "Robust current control-based generalized predictive control with sliding mode disturbance compensation for PMSM drives," ISA Transactions, vol. 71, no. 2, pp. 542-552, 2017.

[10] Y. Deng, J. Wang, H. Li, J. Liu, and D. Tian, “Adaptive sliding mode current control with sliding mode disturbance observer for PMSM drives," ISA Transactions, vol. 88, no. 4, pp. 113-126, 2019.

[11] G. Huang, E. F. Fukushima, J. She, C. Zhang, and J. He, "Estimation of sensor faults and unknown disturbance in current measurement circuits for PMSM drive system," Measurement, vol. 137, pp. 580-587, 2019.

[12] S. H. Ding, J. H. Park, and C. C. Chen, "Second-order sliding mode controller design with output constraint," Automatica, vol. 112, Article ID 108704, 2020.

[13] F.-J. Lin, Y.-C. Hung, and K.-C. Ruan, "An intelligent secondorder sliding-mode control for an electric power steering system using a wavelet fuzzy neural network," IEEE Transactions on Fuzzy Systems, vol. 22, no. 6, pp. 1598-1611, 2014.

[14] W. Xu, Y. Jiang, and C. Mu, "Novel composite sliding mode control for PMSM drive system based on disturbance observer," IEEE Transactions on Applied Superconductivity, vol. 26, no. 7, pp. 12905-12910, 2016.
[15] A. Hosseyni, R. Trabelsi, A. Iqbal et al., "An improved sensorless sliding mode control/adaptive observer of a five-phase permanent magnet synchronous motor drive," International Journal of Advanced Manufacturing Technology, vol. 93, no. 4, pp. 1029-1039, 2017.

[16] Z. Zhou, B. Zhang, and D. Mao, "Robust sliding mode control of PMSM based on rapid nonlinear tracking differentiator and disturbance observer," Sensors, vol. 18, no. 4, pp. 1031-1043, 2018.

[17] K. Mei and S. Ding, "Second-order sliding mode controller design subject to an upper-triangular structure," IEEE Transactions on Systems Man and Cybernetics Systems, vol. 15, pp. 1-11, 2018.

[18] F.-J. Lin, Y.-C. Hung, and M.-T. Tsai, "Fault-tolerant control for six-phase pmsm drive system via intelligent complementary sliding-mode control using TSKFNN-AMF," IEEE Transactions on Industrial Electronics, vol. 60, no. 12, pp. 5747-5762, 2013.

[19] X. Zhao and H. Jin, "Complementary sliding mode control for permanent magnet linear synchronous motor based on elman neural network," Transactions of China Electrotechnical Society, vol. 4, pp. 1-10, 2018.

[20] Y. Huang, W. Huang, S. Chen, and Z. Liu, "Complementary sliding mode control with adaptive switching gain for PMSM," Transactions of the Institute of Measurement and Control, vol. 3, no. 2, pp. 319-3250, 2019.

[21] J. Yan, H. Wang, S. D. Huang, and Y. H. Lan, "Load disturbance observer-based complementary sliding mode control for PMSM of the mine traction electric locomotive," International Journal of Fuzzy Systems, vol. 21, no. 1, pp. 1051-1058, 2019.

[22] B. Zhang, Y. Pi, and Y. Luo, "Fractional order sliding-mode control based on parameters auto-tuning for velocity control of permanent magnet synchronous motor," ISA Transactions, vol. 51, no. 5, pp. 649-656, 2012.

[23] J. Huang, H. Li, Y. Q. Chen et al., "Robust position control of PMSM using fractional-order sliding mode controller," $A b$ stract and Applied Analysis, vol. 2012, no. 4, ArticleID 512703, 33 pages, 2012.

[24] H.-R. Li, Z.-B. Jiang, and N. Kang, "Sliding mode disturbance observer-based fractional second-order nonsingular terminal sliding mode control for PMSM position regulation system," Mathematical Problems in Engineering, vol. 2015, Article ID 370904, 14 pages, 2015.

[25] C.-L. Li and L. Wu, "Sliding mode control for synchronization of fractional permanent magnet synchronous motors with finite time," Optik, vol. 127, no. 6, pp. 3329-3332, 2016.

[26] J. Wang, C. Shao, and Y.-Q. Chen, "Fractional order sliding mode control via disturbance observer for a class of fractional order systems with mismatched disturbance,", Mechatronics, vol. 53, pp. 8-19, 2018.

[27] Q. Hou, S. Ding, and X. Yu, "Composite super-twisting sliding mode control design for PMSM speed regulation problem based on a novel disturbance observer," IEEE Transactions on Energy Conversion, vol. 99, p. 1, 2020.

[28] L. Yu and W. Zhang, "Adaptive non-singular integral terminal sliding mode tracking control for autonomous underwater vehicles," IET Control Theory and Applications, vol. 11, no. 8, pp. 706-710, 2017.

[29] L. Qiao and W. Zhang, "Trajectory tracking control of AUVs via adaptive fast nonsingular integral terminal sliding mode control," IEEE Transactions on Industrial Informatics, vol. 16, no. 2, pp. 1248-1258, 2020. 
[30] W.-H. Chen, "Disturbance observer based control for nonlinear systems," IEEE/ASME Transactions on Mechatronics, vol. 9, no. 4, pp. 706-710, 2004.

[31] I. Podlubny, Fractional Differential Equations, Academie Press, New York, NY, USA, 1999.

[32] D. Matignon, "Stability results on fractional differential equations with applications to control processing," in Proceedings of Computational Engineering in Systems and Application Multiconference Rouen, France, pp. 963-968, 1996.

[33] A. D. Manuel, A. C. Norelys, A. Javier et al., "Using general quadratic Lyapunov functions to prove Lyapunov uniform stability for fractional order systems," Communications in Nonlinear Science and Numerical Simulation, vol. 22, no. 3, pp. 650-659, 2015.

[34] Y. Li, Y. Chen, and I. Podlubny, "Mittag-Leffler stability of fractional order nonlinear dynamic systems," Automatica, vol. 45, no. 8, pp. 1965-1969, 2009. 